\title{
ENSEÑANZA DE LA EPISTEMOLOGÍA DE LA EDUCACIÓN FÍSICA EN EL PROYEC- TO CURRICULAR LICENCIATURA EN EDUCACIÓN FÍSICA DE LA UNIVERSIDAD PEDAGÓGICA NACIONAL: UNA MIRADA DESDE LA HISTORIA.
}

UNA EXPERIENCIA PARA COMPARTIR ${ }^{1}$

EDUCATION OF THE EPISTEMOLOGY OF THE PHYSICAL EDUCATION IN THE PROJECT CURRICULAR LICENTIATE IN PHYSICAL EDUCATION OF THE PEDAGOGIC NATIONAL UNIVERSITY: A LOOK FROM THE HISTORY.

AN EXPERIENCE TO SHARE

Astrid Bibiana Rodríguez Cortés ${ }^{2}$

"La epistemología nació por un descubrimiento Curioso: los científicos se dieron cuenta que el hecho de hacer Ciencia podía no conducir a la ciencia, sino por el contrario

A estar fuera de ella"

Olga Lucía Zuluaga y Humberto Quiceno.

Resumen

Este artículo quiere compartir la experiencia de enseñanza de la epistemología de la educación física, en el Proyecto Curricular Licenciatura en Educación Física de la Universidad Pedagógica Nacional. El texto muestra la manera en que se organizó la propuesta metodológica de una tesis de trabajo, que surge desde discusiones abordadas en el desarrollo de la investigación: Las competencias específicas de la Educación Física factor de calidad de la enseñanza. Dicha propuesta fue implementada en el espacio académico denominado Formación Teórica Disciplinar en los semestres V, VI y VII, entre 2007 y 2008.

Palabras clave: educación física, epistemología, historia, y enseñanza.

Abstract

This article wants to share the experience of education of the epistemology of the Physical Education in the Curricular Project Degree in Physical Education of the National Pedagogical University. The text shows the form as I organize the methodologic proposal of a work thesis, that arises from discussions boarded in the development of the investigation: The specific competitions of the Physical Education quality factor of education. This proposal was implemented in the academic space denominated Theoretical Formation To discipline in the semestres V,VI and VII, between years 2007-2008.

Keywords: physical education, epistemology, history, education

Fecha de recepción: 10 de marzo de 2009.

Fecha de aceptación: 18 de septiembre de 2009.

\section{INTRODUCCIÓN}

\footnotetext{
${ }^{1}$ Este documento es producto de las discusiones y trabajos realizados en la investigación Las competencias específicas de la Educación Física factor de calidad de la enseñanza. CIUP 2007-2008. grupo: Epistemología e Historia de la Educación Física. Línea de investigación: Enseñanza de la Educación Física.

${ }^{2}$ Profesora investigadora auxiliar de tiempo completo de la Universidad Pedagógica Nacional. Licenciada en Educación Física. Magíster en Educación Universidad Pedagógica Nacional. Integrante del Grupo de Investigación Epistemología e Historia de la Educación Física. Correo electrónico: abrodriguez@pedagogica.edu.co - astridbibianarc@yahoo.com.
} 
En primera instancia, se aclara que el contenido de Epistemología de la educación física no se encuentra explícito en la propuesta curricular de la Licenciatura en Educación Física de la Universidad Pedagógica Nacional. Sin embargo, en el segundo ciclo, que es denominado el primer ciclo de profundización, el tema envolvente es la educación física como disciplina académica, ello permite comprender de tajo la apuesta del proyecto curricular por la educación física no como una ciencia, sino como una disciplina, y además de ello una disciplina académica pedagógica, es decir, que estudia, sistematiza y organiza sus conocimientos en la academia. En la parte de contextualización se profundizará sobre la estructura curricular.

La pretensión del primer ciclo de profundización es mirar críticamente cómo la educación física en Colombia se ha consolidado como saber, cuáles han sido los conceptos, las prácticas, las relaciones que ejerce la educación física en los diferentes momentos históricos en los que ha surgido, dejando entrever algunos puntos de quiebre que es necesario investigar, para entender cómo se ha llegado a sistematizar el conocimiento de este saber en algo que se denominara Tendencias actuales de la educación física. En este punto, se intentará analizar algunos problemas actuales a los que se enfrenta la educación física contemporánea, con el fin que el estudiante, al terminar el ciclo, presente su trabajo de sistematización con algunas preguntas que guíen su propuesta curricular particular.

Este documento se encuentra organizado en tres partes: la primera, denominada a modo de contexto, pretende mostrar la organización del Proyecto Curricular Licenciatura en Educación Física de la Universidad Pedagógica Nacional y la forma en que organiza sus ciclos, subproblemas y problemas de área. La segunda parte, tesis de trabajo, muestra el planteamiento teórico sobre el que se basa el desarrollo del espacio académico, Formación Teórico Disciplinar, en el primer ciclo de profundización. La tercera y última parte muestra el desarrollo metodológico de la tesis de trabajo propuesta.

\section{A modo de Contexto}

De manera sucinta se pretende mostrar la estructura del Proyecto Curricular Licenciatura en Educa- ción Física (PC-LEF). En primera instancia, este Proyecto Curricular asume las directrices académicas de la Universidad Pedagógica Nacional, con una tendencia curricular transformacionista y de currículo integrado. Los principios que orientan su desarrollo son: integración de saberes, democracia, autonomía, ética y principio contextual.

Cuenta con una macroestructura, en la que se encuentran ubicados los ciclos, ambientes, campos de formación y áreas académicas. Los ciclos de formación, denominados así porque son la unidad temporal que organiza los grados de profundización o complejidad del conocimiento, en torno a unos saberes que se interrelacionan en el plan de estudios.

El documento de Condiciones Iniciales de la Licenciatura en Educación Física (2004), aclara los propósitos de los ciclos y su intencionalidad: el primer ciclo es denominado ciclo de fundamentación, comprende los cuatro primeros semestres, su propósito es la aproximación al análisis y comprensión de los universales, que fundamentan la construcción del conocimiento y su relación con la profesión educativa. El segundo ciclo, denominado ciclo de profundización, comprende desde el semestre cinco hasta el diez y está compuesto por dos niveles: el primer nivel contempla la construcción disciplinar de la educación física y se cursa en los semestres $\mathrm{V}$, VI y VII y tiene como propósito hacer una aplicación crítica de principios universales, tal, que sirva como fundamento de análisis y construcción del saber disciplinar de la educación física. Al segundo nivel le corresponde la proyección social de la educación física que se cursa en los semestres VIII, IX y X. Tiene como propósito dialogar, debatir y difundir sometiendo a juicio crítico de la comunidad en general, la propuesta humanística-pedagógica y disciplinar diseñada por el futuro profesional y expresada en un proyecto curricular particular.

Las áreas entendidas como la unidad estructural académica son: área humanística, área pedagógica y área disciplinar. Cada área se compone de una formación teórica (disciplinar, pedagógica y humanística), que tiene como fin la fundamentación sobre las que se construyen las concepciones de educación física y un taller (taller de experiencias corporales, taller de cuerpo, taller de lenguajes y taller de confrontación) los que conciben al cuerpo como el mediador entre el sujeto y el mundo, realizando una 
reflexión constante sobre esta mediación.

El Proyecto Curricular centra su preocupación en desarrollar jerarquías de procesos de pensamiento, como son: descripción, explicación, comprensión y relación, conduciendo con ello a formar estudiantes críticos. Ante esta Perspectiva, el maestro que orienta los espacios en el Proyecto Curricular, tiene la flexibilidad de organizar su propuesta temática, pero, al mismo tiempo, tiene la responsabilidad de orientar el desarrollo de un ciclo, el que tiene unas implicaciones en la formación de futuros maestros de educación física.

Este estudio se encuentra ubicado en el segundo ciclo, primer nivel de profundización semestres V, VI, VII, en el área disciplinar. El tema envolvente de este ciclo es la educación física como disciplina académica. Los ejes de análisis del área disciplinar son: Experiencias corporales y Procesos del desarrollo del potencial humano.

El núcleo temático es técnicas del movimiento corporal. El tema envolvente, los ejes de análisis y el núcleo temático guían y enfocan los procesos de programación de los espacios académicos del área, entendiendo que el área se compone del espacio de formación teórico disciplinar y el taller de cuerpo. Por ello, se expondrá y develará los procesos de apropiación, apuestas teóricas y metodológicas que se hace en la implementación de esta propuesta académica.

\section{TESIS DE TRABAJO}

En el proceso de formación de los futuros licenciados en educación física, es preciso mirar con detenimiento los procesos de instauración de los discursos de la educación física en Colombia, por ello la tesis que sustenta la propuesta académica es: Realizar la arqueología de los discursos que han constituido el saber de la educación física en Colombia posibilita estructurar una epistemología de este saber.

Se empezará por aclarar qué se entiende por Epistemología y la concepción sobre saber. La epistemología se concibe

Como la disciplina que se ocupa de estudiar la forma como se ha producido y reproducido el saber en una disciplina ó campo de análisis. Es necesario entender que esa producción tiene que ver con unos momentos históricos determinados y por ello existen unos modos y condiciones de apropiación diversas" (Salazar, 1998, p. iii).

Se asume por saber "un conjunto de conocimientos metódicamente producidos que puedan transmitirse mediante un proceso pedagógico de enseñanza" (Salazar, 1998, p. iii). Japiassu (citado por Salazar, 1998) comenta que esta noción no obstante merece una explicación. Dos elementos concurren en el saber: por un lado, la producción metódica de conocimientos; por otro, su reproducción metódica con fines pedagógicos y de enseñanza. Esta manera de acercarse a definir lo que es epistemología y saber llevar a pensar, en una epistemología que usa como procedimiento teórico metodológico, la arqueología en un saber como la educación física.

\section{UNA MIRADA DESDE LA ARQUEOLOGÍA}

Para dar estructura a la tesis propuesta se partirá de la arqueología en una mirada desde la epistemología. Roberto Salazar (1998) hace un estudio de las diferentes clases de epistemologías a partir de cuatro tendencias, en las que es posible analizar el pensamiento científico: epistemologías empíricoanalíticas, epistemologías crítico-racionalistas, epistemologías crítico-sociales y epistemologías histórico-genéticas. En esta clasificación se encuentra la propuesta de trabajo situada en las epistemologías histórico-genéticas.

Las epistemologías histórico-genéticas, basan sus estudios en la historia, en este caso la historia del saber de la educación física, centrándose en la determinación de la lógica de su emergencia y desarrollo. Pero, no se trata de hacer cualquier clase de historia, por ejemplo: no es una historia apologética o crónica, lo que se pretende hacer es mirar como la educación física se ha estructurado como una disciplina, es decir, con sistemas culturales, procesos sociales de búsqueda de intereses, de intenciones y de rupturas.

Uno de los filósofos que incluye Salazar (1998) como parte de la epistemologías histórico-genéticas, es a Michel Foucault. Desde la perspectiva foucaultiana, la historia pretende romper con la mirada un hilo conductor, con la explicación lineal de los procesos sociales. Foucault propone que la,

Arqueología, más que determinar continuidades, se orienta a la identificación o establecimiento de las discontinuidades del saber y, en especial, en los 
umbrales en que todavía no es posible definir, si el saber se positiviza o no. Sus pretensiones no han sido la de constituirse en epistemólogo, ni sus trabajos pueden ser calificados como tales. Muestra, en cambio, los procesos o dispositivos a partir de los que se entreteje el saber y el poder"(1998, p. 163).

Salazar (1998) explica que la arqueología no es estrictamente una epistemología, por cuanto ella, no tiene como objetivo mostrar los procesos a partir de los que una disciplina atraviesa el umbral de su positividad, ni mostrar los elementos que la harían mantenerse en el ámbito de la cientificidad. Tampoco, tiene como objetivo analizar los procesos de estructuración de sus métodos, ni de la lógica a partir de la que se configuran, ni de probar la validez o no de sus enunciados, ni de la objetividad que alcanzan como discursos científicos.

Las pretensiones de realizar un acercamiento desde la arqueología de la educación física en Colombia con pretensiones epistemológicas rompen con la perspectiva de mirar la educación física como una ciencia que ni siquiera está en construcción, pero, en este sentido, sí tiene grandes implicaciones de mirar con cuidado la carrera emprendida en algunos momentos históricos, por plegarse a las ciencias naturales para ingresar a la cientificidad, así como el viraje que ha tenido en los últimos años para hacer más cercano su saber a las ciencias humanas, quizá con el fin de encontrar un reconocimiento como disciplina social.

Salazar (1998) muestra como desde Foucault la episteme puede entenderse como la infraestructura cultural del saber, aquello que está en la base de la constitución del conocimiento común y de las ciencias. En este sentido, reconocer que el saber de la educación física se ha constituido en unas técnicas, unas prácticas, unos lenguajes, unas percepciones sociales y unas propuestas teóricas que entra a formar parte de la episteme, es decir, de la infraestructura del saber cultural de la educación física.

Por ello, el análisis y construcción de un conocimiento de la educación física busca la consolidación y organización de unos conocimientos, busca marcar una territorialidad estableciendo unas estructuras de saber, poder y verdad, características de las disciplinas contemporáneas. Desde esta perspectiva, la educación física sigue en su indagación por convertirse en una disciplina cada vez más rigurosa. Sin embargo, es posible considerar que no existe una sola forma de hablar de epistemología de la educación física, y más aún, si se entiende por epistemología, sólo aquella disciplina que se encarga de hablar de los conocimientos científicos.

En este texto pretende plantearse que el concepto de epistemología no se mueve en la rigidez que pretende la ciencia, se acepta la epistemología como una disciplina que muestra la consolidación de los saberes. Por lo tanto, se plantea hablar de una episteme del saber de la Educación Física. Considerando que al reconocérsela de esta manera, podríamos dar validez a la multiplicidad de conocimientos construidos en el campo de estudio, asimismo, reconocer la importancia que ha tenido el conocimiento y la práctica cotidiana en la construcción del saber de esta disciplina.

Al estudiar con cuidado la arqueología de la educación física en Colombia, se busca dar respuesta a las siguientes preguntas: ¿cómo se ha constituido ${ }^{3}$ el saber de la educación física en Colombia?, ¿cuáles han sido las condiciones de posibilidad que han permitido emerger las diferentes tendencias de la educación física en Colombia?, en este sentido, no se trata de hacer la historia de la educación física en Colombia, se pretende comprender las condiciones de posibilidad ${ }^{4}$, cómo han emergido los diferentes discursos, que existen sobre el saber de la educación física, así como mirar los diferentes objetos de estudio y analizar en relación con los momentos históricos y sociales, en los que han surgido, así como, estudiar los elementos del campo de la educación que han permeado la Educación Física.

Esta tesis de trabajo se encuentra en construcción y análisis, lleva algunos años de exploración, desde

3 Es importante retomar la claridad que hace Germán Vargas Guillén (2006) cuando muestra el tránsito sugerido entre la constitución y la construcción del conocimiento. "Haciendo énfasis en que la donación absoluta o la conciencia vivida, en actitud natural del objeto o la experiencia vivida cabe discutir desde el contexto de la construcción, sino desde la constitución objetiva del conocimiento".

4 En este sentido Alejandro Álvarez hace una precisión: "Si la arqueología identifica las huellas, es decir, la forma de existencia de unas prácticas, la genealogía identifica las condiciones que las hicieron posibles, es decir, sus condiciones de existencia". 
los trabajos de investigación, pasando por el análisis de diversas fuentes que permitan comprender para qué, desde dónde y cómo repensar los procesos de enseñanza de la epistemología de la educación física, en los futuros licenciados.

Desde la enseñanza, se plantea cómo no ofrecer simplemente un curso de teorías sueltas, por ello la metodología de desarrollo de la tesis de trabajo expuesta aquí parte de pensar que no se trata de resolver problemas, sino de crearlos para permitir a los estudiantes determinar la ambigüedad, diversidad y necesidad de usar elementos de la investigación para comprender, el intrincado campo de estudio que es la educación física.

Sin embargo, como lo dice Martín Heidegger: "Enseñar es más difícil que aprender", por ello, en la preparación, planeación y puesta en escena de esta tesis, se ha pasado desde el humor al sarcasmo, pasando por la confusión y quizá la incertidumbre, intentar justificar un proceso arqueológico de la Educación Física en Colombia, es necesario para entender la consolidación de esta disciplina en el presente; no es tan sencillo, en algunos casos por la manera misma en que se ha estructurado el saber disciplinar.

Este documento es un primer avance sobre el análisis del proceso de enseñanza del tema envolvente: la educación física como disciplina académica, pero, queda por hacer el análisis, desde el aprendizaje, siendo este aspecto vital e importante para retroalimentar y conversar sobre las apuestas que hace un maestro, cuando planea un nuevo curso.

\section{UNA PROPUESTA METODOLÓGICA, UNA PRO- PUESTA TEÓRICA O UNA CAJA DE HERRAMIEN- TAS $^{5}$}

\section{El caso del Primer Ciclo de Profundización en el área disciplinar en 2007-2008.}

Este apartado pretende mostrar el proceso de desarrollo de la tesis de trabajo en el periodo com-

\footnotetext{
5 Alejandro Álvarez en su libro "La escuela se hizo necesaria", muestra el debate por la categoría propuesta por Foucault, en relación con entender la genealogía y la arqueología como un enfoque metodológico, una teoría o como una caja de herramientas.
}

prendido entre 2007 y 2008, se busca compartir la metodología, los conceptos, las fuentes de consulta, las inquietudes y percepciones, frente al desarrollo de este espacio de encuentro académico.

El espacio se desarrolla en tres semestres, comprendidos entre el V, VI y VII semestre. El propósito del V semestre, es realizar una genealogía del concepto de educación física en Colombia, mostrar la metodología arqueológica-genealógica en algunos trabajos investigativos y conceptualizar la apuesta teórica de la concepción de epistemología.

Para dar desarrollo al propósito en $\mathrm{V}$ semestre, se emplearon unas fuentes de consulta que se denominaron secundarias, por el uso que le darían los estudiantes en su proceso de sistematización del ciclo (trabajo final del ciclo). Las fuentes seleccionadas fueron organizadas de manera cronológica, para permitir al estudiante observar la genealogía del concepto de educación física, pero, haciendo énfasis en que el tiempo no era el único criterio en la forma de hacer la historia y en el desarrollo metodológico de las investigaciones.

Desde el estudio de los documentos que pueden ayudar a comprender la investigación arqueológica, se pueden abordar los trabajos del grupo de las prácticas pedagógicas y sus diferentes investigaciones en el campo educativo. Sin embargo, emprender la investigación arqueológica de la educación física requiere mirar diversos elementos y trabajos que se han realizado desde este enfoque teórico-metodológico, algunos de estos trabajos son citados en el desarrollo de este escrito y son insumo importante para la comprensión de lo expuesto.

El primer documento que se decide abordar, es el trabajo de Miguel Vicente Pedraz (2008), este autor, aunque no hace una investigación tomando como referencia el caso colombiano, permite mostrar a los estudiantes tres aspectos importantes: la posición personal del autor frente al concepto de educación física, el que devela cómo en un contexto social determinado se da la configuración de este concepto, sus prácticas e imaginarios. El segundo elemento es el uso de la arqueología en el desarrollo de su trabajo y, determinante en la metodología de aplicación y el tercer aspecto es el surgimiento de lo que denominaría los objetos fundantes de la educación física: cuerpo, juego y movimiento. 
Una vez estudiada la apuesta metodológica del curso, se abordan las fuentes investigativas que toman como objeto de estudio el desarrollo de la educación física en Colombia. Para ello, se empieza con el trabajo realizado por la Universidad de Antioquía: Discursos de la educación física de siglo XIX en Medellín, este texto muestra las denominaciones, prácticas, métodos de enseñanza de los discursos de la Educación Física en el Siglo XIX, una manera de hacer historia, esta investigación, de forma particular, enseña a los estudiantes lo que significa el tiempo en la historia, no sólo como medida de organización, sino, como fue denominado por los investigadores, "desparramiento temporal", elemento fundamental para mostrar la manera de categorizar y dejar hablar las fuentes, aspecto que se desarrollaría con profundidad en un taller práctico, que se comentará más adelante.

Otro documento de estudio son los lineamientos curriculares en educación física, (MEN, 2000) en el capítulo de referentes conceptuales, se hace un acercamiento al concepto de educación física en Colombia, en la segunda mitad del siglo XIX. En este texto, de manera cronológica, se organiza lo que los autores denominan la "evolución histórica de la educación física escolar". Este capítulo muestra a los estudiantes, además de las fuentes primarias citadas con conceptos de educación física, otra concepción sobre la historia, otra forma de hacer la historia. En este texto, el tiempo es una línea en la que los acontecimientos transcurren uno tras otro, no da pie para observar las fracturas, ni las discontinuidades en los hechos, tiene como objetivo develar cómo ha sido el proceso de consolidación de la educación física en la escuela colombiana. Este texto permite mostrar otra forma de analizar las fuentes primarias y una vía distinta de hacer historia; quizá desde la contrastación, el estudiante puede comprender los aspectos que se han venido abordando.

Otro texto de consulta, que accede a mirar el papel de la educación física en la primera mitad del siglo XX, es el trabajo realizado por Javier Sáenz, Óscar Saldarriaga y Armando Ospina (1997), Mirar la infancia: pedagogía, moral y modernidad en Colombia, 1903-1946. Este trabajo muestra la importancia de la educación física en la escuela colombiana en los primeros años del siglo XX, su relación con la pedagogía activa, su rápida articulación con el dis- curso médico, la vigorización fisiológica de la raza y su importancia en los nuevos imaginarios, discursos y prácticas de la época sobre el cuerpo, la moral y la inteligencia de los niños. Estos autores, con un trabajo muy juicioso y riguroso, exponen cómo se entrecruzan las fuerzas, saberes y poderes para producir maneras de apropiación del discurso modernista en Colombia. Además de ser, un trabajo en que se puede apreciar la importancia de la educación física en un momento histórico determinado, cómo se habla sobre ella, cómo se legisla y qué relevancia tiene para el Estado del momento. De manera metodológica, este trabajo permite a los estudiantes establecer relaciones entre lo pedagógico, lo humanista y lo disciplinar.

Otras fuentes secundarias de consulta son Las Prácticas Corporales y la educación física en la escuela primaria en Colombia entre 1870 y 1913 por Ximena Herrera (2000), educación física en el proceso de modernización por Víctor Chinchilla (2002) y Las prácticas corporales y su relación con la pedagogía en la escuela colombiana en 1935 por Astrid Bibiana Rodríguez (2006). Estos textos, entre otros trabajos de corte histórico consultados en la clase, permiten que los estudiantes revisen distintos objetos de investigación, desde la perspectiva histórica sobre la educación física en la primera mitad del siglo XX, observen las metodologías de investigación y los resultados alcanzados en cada una de ellas.

Para complementar el trabajo de lectura de las fuentes secundarias, se hace una invitación a los autores de algunas de las investigaciones abordadas al espacio de encuentro académico, desde la metodología de conversatorio, se resuelven algunas inquietudes que los estudiantes tienen sobre lo metodológico, fundamentos teóricos (consolidación de la educación física en el momento histórico abordado), y resultados de la investigación, así como, la importancia de la investigación histórica para el campo de estudio de la educación física.

\section{Taller práctico}

Simultáneo al trabajo de lectura de fuentes secundarias, se realiza un taller práctico denominado: Hacer Historia de la Educación Física, el objetivo de este es acercar a los estudiantes a fuentes primarias, que muestren conceptos de Educación Física en un contexto social determinado. 
El taller consiste en que los estudiantes consulten fuentes primarias seleccionadas entre 1900 y 1950. Estas fuentes han sido previamente revisadas por el maestro de la clase, que orienta a sus estudiantes sobre el tipo de fuente, la contextualización de ésta y la periodicidad. Las fuentes primarias seleccionadas son: revista Cromos, periódico el Tiempo, revista Semana, revista Iglesia, periódico El Estudiante. Mediante una guía, se le explica a los estudiantes que tenga en cuenta, los siguientes ejes para hacer la revisión y selección de los artículos a tematizar: educación, educación física, cultura física, deporte y cuerpo $^{6}$. Los estudiantes seleccionan los conceptos que textualmente se muestran en las fuentes y los organizan por los ejes temáticos solicitados. Una vez organizados los artículos seleccionados, el estudiante escoge un eje de análisis y en un documento escrito establece las relaciones entre las fuentes primarias, las secundarias y el contexto histórico, social, político, educativo y cultural.

Como productos de este ejercicio, algunos estudiantes logran hacer escritos interesantes, con temas como: la relación entre el movimiento racial y eugenésico y la educación física, los imaginarios de cuerpo y belleza en el contexto histórico estudiado, el desarrollo de la educación física escolar en instituciones, la presencia de los discursos morales en la construcción de cuerpo y el acercamiento a la historia de algunas disciplinas deportivas en Colombia como: sóftbol, fútbol, boxeo y lucha, entre otros. Esta clasificación de los trabajos presentados por los estudiantes permite retroalimentar algunos aspectos de carácter metodológico, asimismo, el impacto, la influencia de algunos temas o el interés de los estudiantes por algún eje de análisis.

Una de las fortalezas que enuncian los estudiantes de la realización de este taller es la dificultad en la constitución del saber disciplinar, el agenciamiento de diversas instituciones frente a los imaginarios de belleza y cuerpo, evidencian la diversidad de los discursos entorno a la educación física. Algunas dificultades que los estudiantes enumeran es el acceso a los materiales, la relación de estos discursos con la didáctica de la educación física y el análisis de la

\footnotetext{
6 Los ejes seleccionados han sido previamente trabajados en la clase desde las fuentes secundarias, son referentes que guían las investigaciones de forma transversal.
}

información.

Al terminar este ejercicio de investigación formativa, en el semestre VI, se hace una socialización que tiene como fin contextualizar la Educación Física, en la primera década del siglo XX, determinando en Colombia los saberes que construyen y constituyen el saber disciplinar.

TENDENCIAS DE LA EDUCACIÓN FÍSICA: UNA MIRADA AL PRESENTE

Entrando al semestre VII, en el que se presenta el trabajo de sistematización, se aborda la década de los sesenta, que se denominó el momento expansivo de la educación física, algunos acontecimientos como: la apertura de la Universidad Pedagógica Nacional y el primer programa de la Universidad en la Facultad de Educación Física en 1962, el surgimiento de Coldeportes en 1967 y, con ello, gran parte de la legislación deportiva, la entrada de un gran número de libros en educación física, deporte, gimnasia, la consolidación de la educación física en la escuela colombiana, la llegada de algunos maestros extranjeros con nuevos discursos y prácticas. Sin embargo, no se encuentran muchos trabajos investigativos dedicados al estudio de estas décadas, los que no permite entender ¿cómo han sido los procesos de apropiación discursiva que llevaron a sistematizar el conocimiento de la educación física en lo que se ha denominado tendencias de la educación física en Colombia?

Para abordar este tema, se asume la propuesta realizada por el grupo de investigación "Filia"7 de la Universidad Pedagógica Nacional, que plantea: abordar desde el enfoque de las ciencias sociales los paradigmas que han fundamentado las diversas teorías de la educación física. En esa búsqueda, encontraron cinco paradigmas: la condición física, el deporte, la psicomotricidad, la expresión corporal y la praxiología motriz; que suelen identificarse como tendencias, enfoques, corrientes o inclusive como contenidos de la educación física.

El propósito de estudiar las tendencias de la Educación Física, en el VII semestre es abordar la teoría

El grupo de investigación Filia está compuesto por: Libardo Mosquera Mateus, Henry Mauricio Ortega Camacho, Édgar Mauricio Robayo y Rubén Darío Torres. 
producida en el campo, organizarla y sistematizarla, a partir de cuatro preguntas: 1. ¿cuál es el objeto de estudio que propone el autor?, 2. ¿cuáles son los principales conceptos y metodologías investigativas del autor?, 3. ¿qué concepto de educación física propone el autor? 4. ¿qué pedagogía y didáctica aborda?. Metodológicamente, se continúa con la misma perspectiva genealógica-arqueológica.

En procura de desarrollar el tema de las tendencias de la Educación Física desde las preguntas planteadas se realizan tres acciones: la primera acción es seleccionar los autores y sus obras más representativas, desde las que se pretende dar respuesta a las cuatro preguntas planteadas. La segunda acción es la lectura de fuentes secundarias e investigaciones contemporáneas sobre cada tendencia. La tercera acción es un ejercicio práctico de aplicación pedagógica y didáctica de la tendencia de estudio.

Una vez desarrolladas las tres acciones propuestas, se realiza el trabajo final del ciclo denominado sistematización, para la realización de este ejercicio investigativo de aula, los estudiantes agrupan los trabajos realizados a lo largo del ciclo, organizan la información y centran su esfuerzo en dar respuesta a las preguntas propuestas al empezar el ciclo: ¿cómo se ha constituido el saber de la educación física en Colombia?, ¿cuáles han sido las condiciones de posibilidad que han permitido emerger las diferentes tendencias de la Educación Física en Colombia? La respuesta a estas preguntas se realiza desde el estudio juicioso de una de las tendencias de la educación física, que escoge el estudiante desde su preferencia.

En las sistematizaciones, entendiendo por ellas, como lo dicen Lola Cendales y Alfonso Torres, una forma de recuperar los saberes y significados de la experiencia para potenciarla, se han presentado trabajos que llegan a hacer acercamientos pertinentes, al presente con una mirada reflexiva y cuestionadora de las prácticas y discursos que, constituyen actualmente la realidad de la educación física.

Los trabajos de sistematización han permitido encontrar que se necesita más tiempo para cumplir con los objetivos propuestos, en algunos casos, no se logra relacionar el tema del taller práctico en la búsqueda de la fuente primaria, con las tendencias contemporáneas de la educación física, sin embargo, otros estudiantes muestran trabajos que intentan responder, las preguntas planteadas como desarrollo temático deseando relacionar lo visto en el primer ciclo de profundización en el espacio teórico disciplinar.

\section{REFLEXIONES FINALES}

Lo primero que se puede decir es que este es un trabajo sin conclusión, puesto que la propuesta es parte de un ejercicio investigativo constante y, por tanto, aún no está terminado. La escritura de este documento permitió un encuentro de autoreflexión; en ese sentido, lo que se puede expresar a continuación son unas sencillas consideraciones.

La implementación de esta, aún nueva propuesta curricular de Licenciatura en Educación Física que hace la Universidad Pedagógica Nacional me ha permitido enriquecer mi experiencia personal y profesional, porque ha sido un constante devenir, encontrar unas apuestas académicas y poderlas llevar a cabo, es una posibilidad, pero, también, es una responsabilidad y un esfuerzo por transmitir e impregnar a los estudiantes de una forma de acercarse al conocimiento y percibir la vida.

Intentar mostrar la importancia del estudio histórico, de una disciplina como la educación física, que aún presenta dificultad, porque se pretende resolver los problemas de la práctica con la práctica misma, en algunos casos no se le da validez al estudio del medio, ni del contexto histórico como construcción de realidades, la pregunta por el sujeto y su construcción social no parece ser determinante, cuando se intenta controlar las masas con las técnicas de movimiento, en este sentido, el estudio de la técnica como producción sigue presentándose para algunos estudiantes como el deber ser de la educación física y ello merma sentido a la discusión por la praxis del saber.

Abordar un contenido como la epistemología de la Educación Física en Colombia requiere de un trabajo de investigación permanente que nos permita comprender cómo la teoría del campo, hoy sistematizada en tendencias de la educación física surgió, y llegó a ser apropiada, por algunos maestros en su quehacer. En este sentido, es válido reconocer, el saber de los primeros maestros y maestras de la Universidad Pedagógica Nacional, pues ellos son ac- 
tores determinantes en las prácticas discursivas de los maestros contemporáneos, así como en la construcción del campo disciplinar.

Por ello, la discusión y la reflexión en el grupo de investigación de Epistemología e Historia de la Educación Física pretende no solamente fortalecer la claridad conceptual, sino el desarrollo profesional que proyecte a los nuevos licenciados en educación física, los que requerirán hacer un análisis conceptual, contextual, e histórico, para lograr establecer conexiones certeras con los nuevos educandos. Asimismo, el grupo de investigación pretende establecer: qué le compete a la educación física desde lo epistemológico, lo práxico y lo social.

Sea este el momento para agradecer a los estudiantes que compartieron el desarrollo del espacio (a que se hace referencia) de encuentro académico Formación Teórico Disciplinar entre 2007 y 2008, porque ellos me impulsaron a sistematizar esta propuesta de enseñanza, la que al final del ciclo pudimos evaluar, usando la conversación como pretexto; sin embargo, puedo plantear que fue sencillamente seguir comprendiendo cómo los procesos de enseñanza son una determinación y una apuesta del maestro para compartir una parte de lo que él puede ser.

\section{REFERENCIAS BIBLIOGRÁFICAS}

Álvarez, A. (1994). La escuela se hizo necesaria. Bogotá: Magisterio.

Chinchilla, G. V. (2002). Educación física en el proceso de modernización. Revista Lúdica, 7, 3-17.

Facultad de Educación Física de la Universidad Pedagógica Nacional.(2004). Proyecto Curricular en Educación Física. Documento de Condiciones Iníciales, Bogotá: Universidad Pedagógica Nacional.

García, C. Franco, S. García, F. Vásquez, C. y Urrego, L.(2000). Recuperación documental de los discursos acerca de la educación física del siglo XIX en Medellín. Medellín: Universidad de Antioquia.

Herrera, C. (2000). Las prácticas corporales y la Educación Física en la escuela primaria en Colombia entre 1870 y 1913. Revista Lúdica Pedagógica. 4, 3-14.

Ministerio de Educación Nacional. (2000). Lineamientos Curriculares en Educación Física. Bogotá: Ministerio de Educación Nacional.

Mosquera, L., Robayo, M., Ortega M. y Torres, R. (2005). Paradigmas y Ejes de la Educación Física. Bogotá: Universidad Pedagógica Nacional. I
Pedraz, V. (2008). Arqueología de la Educación Física y Otros Ensayos. Bogotá: Kinesis.

Rodríguez, C. A. (2006). Las Prácticas Corporales y su Relación con la Pedagogía en la Escuela Colombiana en 1935. Revista Lúdica 11, 132-138.

Sáenz, J., Saldarriaga, O. y Ospina A. (1997). Mirar la Infancia: Pedagogía, Moral y modernidad en Colombia, 1903-1946. Medellín: Universidad de Antioquia.

Salazar, R. (1998). Introducción a la epistemología. Bogotá: Unad.

Vargas Guillén, G. (2006). Tratado de epistemología. Bogotá: Universidad Pedagógica Nacional-San Pablo. 